\title{
COSTA DE MANGUEZAIS DE MACROMARÉ DA AMAZÔNIA: CENÁRIOS MORFOLÓGICOS, MAPEAMENTO E QUANTIFICAÇÃO DE ÁREAS USANDO DADOS DE SENSORES REMOTOS
}

\author{
Pedro Walfir Martins Souza Filho \\ Recebido em 23 setembro, 2005 / Aceito em 27 dezembro, 2005 \\ Received on September 23, 2005 / Accepted on December 27, 2005
}

\begin{abstract}
According to the World Mangrove Atlas, this ecosystem represents $8 \%$ of the world's coastline and one quarter of the tropical coastal zone shoreline, embracing $181,077 \mathrm{~km}^{2}$. The aim of this work is to map and to quantify the extension of macrotidal mangroves along the northeastern Pará and northwestern Maranhão coasts, here named as Amazon Macrotidal Mangrove Coast (AMMC). The identification of mangrove areas and their quantification were carried out using Landsat-7 ETM $^{+}$images acquired in 1999 and 2000 and a geographic information system (GIS). The integration of remote sensing data, geological and oceanographic data allowed the recognition of five geomorphologic sectors, representing a total mangroves area of $7.591 \mathrm{~km}^{2}$. This area represents the largest continuous mangrove belt in the world and constitutes $56,6 \%$ of mangroves in Brazil. Prioritary measures for Amazon mangrove conservation must be taken and research to enhance understanding of this complex and important ecosystem must be supported and developed.
\end{abstract}

Keywords: mangroves, area estimative, remote sensing, Amazon.

RESUMO. De acordo com o Atlas de Manguezais, este ecossistema representa $8 \%$ de toda a linha de costa do planeta e um quarto da linha de costa da zona tropical, perfazendo um total de $181.077 \mathrm{~km}^{2}$. Este trabalho objetiva quantificar a extensão dos manguezais de macromaré da costa nordeste do Pará e noroeste do Maranhão, aqui denominada de Costa de Manguezais de Macromaré da Amazônia (CMMA). 0 reconhecimento das áreas de manguezais e sua quantificação foram realizados a partir da utilização de imagens Landsat-7 ETM+ ${ }^{+}$, adquiridas em 1999 e 2000 e de um sistema de informações geográficas (SIG). A integração dos dados de sensores remotos, dados geológicos e oceanográficos permitiu o reconhecimento de cinco setores geomorfológicos, que abrangem uma superfície total de $7.591 \mathrm{~km}^{2}$ de manguezais. Esta área representa a maior faixa de manguezais contínuos do planeta e corresponde a $56,6 \%$ dos manguezais do Brasil. Medidas prioritárias de conservação dos manguezais da Amazônia devem ser tomadas e pesquisas que busquem a melhor compreensão deste complexo e importante ecossistema devem ser financiadas e desenvolvidas.

Palavras-chave: manguezais, estimativa de área, sensoriamento remoto, Amazônia.

Universidade Federal do Pará, Centro de Geociências, Laboratório de Análise de Imagens do Trópico Úmido. Av. Augusto Correa 1, Campus do Guamá, Caixa Postal 8608, 66075-110 Belém, Pará, Brasil. Fone: (+55 91) 3201-8009; Fax: (+55 91) 3183-1478 -E-mail: walfir@ufpa.br 


\section{INTRODUÇÃo}

Existe uma considerável quantidade de informações disponíveis sobre a distribuição de manguezais ao longo da zona costeira de todo o mundo. De acordo com o Atlas de Manguezais (Spalding et al., 1997), este ecossistema representa $8 \%$ de toda a linha de costa do planeta e um quarto da linha de costa da zona tropical, perfazendo um total de $181.077 \mathrm{~km}^{2}$. Vale ressaltar que o Brasil é o segundo país em extensão de áreas de manguezal $\left(13.400 \mathrm{~km}^{2}\right)$, ficando atrás apenas da Indonésia, que apresenta $42.550 \mathrm{~km}^{2}$, distribuídos ao longo de seus arquipélagos (Spalding et al., 1997). Nos últimos anos, o mapeamento dos manguezais tem sido realizado amplamente em todo o mundo, retratando não somente a hidrografia, topografia, vegetação e geomorfologia, mas também inventários de recursos naturais, planos de gerenciamento da zona costeira, mapas das zonas úmidas e mapas de sensibilidade costeira produzidos em escalas nacionais, regionais e locais. Alguns projetos de mapeamento de manguezais já foram desenvolvidos em todo o mundo (Clough, 1993; Diop, 1993; Lacerda, 1993; Prost, 1997).

No Brasil, estudos sobre o mapeamento, distribuição e variabilidade das áreas de manguezal vêm sendo realizados desde a década de 80, com destaque para os trabalhos de SchaefferNovelli (1989), Schaeffer-Novelli et al. (1990), Herz (1991), Franzinelli (1992), Kjerfve \& Lacerda (1993), Rebelo-Mochel (1997), Schaeffer-Novelli \& Cintrón-Molero (1999) e Kjerfve et al. (2002). Os manguezais de macromaré da costa nordeste do Pará e noroeste do Maranhão, aqui denominada de Costa de Manguezais de Macromaré da Amazônia - CMMA (Fig. 1), estende-se da Baía de Marajó (PA) até a Ponta de Tubarão, Baía de São José (MA), perfazendo cerca de $650 \mathrm{~km}$ de litoral em linha reta.

Esta área é caracterizada por um relevo baixo ( 0 a $80 \mathrm{~m}$ ), ampla planície costeira (com até $70 \mathrm{~km}$ de largura) e extensa plataforma continental adjacente ( $200 \mathrm{~km}$ de largura), sendo extremamente irregular, endentada e recortada por vários estuários. A CMMA está sujeita a um regime de macromarés semidiurnas, com variações em torno de $4 \mathrm{~m}$ na Baía de Guajará, em Belém e 7,5 m na Baía de São Marcos (DHN, 2004). 0 clima é quente e úmido, com estação seca (julho a dezembro) e chuvosa (janeiro a maio) bem definidas, com precipitação média anual variando de 2500 a $3000 \mathrm{~mm}$ anuais e temperatura média em torno de $26^{\circ} \mathrm{C}$ (Moraes et al., 2005).

Neste trabalho, o mapeamento dos manguezais está fundamentado na utilização de imagens do sensor ETM Landsat-7 (pixel reamostrado para $90 \mathrm{~m}$ ) e de um sistema de informações geográficas (SIG). 0 mapeamento restringiu-se apenas à floresta de manguezal, com o objetivo de mapear e quantificar tal ecossistema influenciado por um regime de macromaré ao longo da costa amazônica. Esta região é definida aqui como o maior cinturão contínuo de manguezais do planeta.

\section{MAPEAMENTO DE MANGUEZAIS}

Diversos trabalhos foram realizados com a finalidade de mapear e quantificar a área total do planeta ocupada por manguezais. As Tabelas 1 e 2 sintetizam a estimativa de áreas de manguezal ao longo de todo o globo, com o percentual de áreas por continente e pelos sete países com maior extensão de área de manguezais, respectivamente. Os valores obtidos, apesar de relativamente diferentes, nos fornecem uma razoável estimativa da área total de manguezais no mundo (Spalding et al., 1997). Este problema no cálculo das áreas está associado às diferentes metodologias empregadas, que incluem fontes de dados com resoluções espaciais diversificadas, bem como porções dos diferentes ecossistemas costeiros adjacentes (e.g. pântanos, planícies de marés, planícies de inundação fluvial).

\section{MATERIAIS E MÉTODOS}

0 reconhecimento das áreas de manguezais e sua quantificação foram realizados a partir da utilização de imagens Landsat-7 $\mathrm{ETM}^{+}$, adquiridas em 1999 e 2000. As bandas 3, 4 e 5 foram processadas, com sua geometria corrigida a partir de pontos de controle coletados em campo com GPS e com cartas planialtimétricas produzidas a partir do processamento de dados do SRTM - Shuttle Radar Topographic Mission (Rabus et al., 2003). 0 pixel original das imagens Landsat foi reamostrado pelo método de vizinho mais próximo para $90 \mathrm{~m}$, bem como georreferenciado para 0 sistema de coordenadas geográficas (latitude e longitude) no Datum WGS 84. Além disso foi aplicada a técnica de realce linear de contraste. Posteriormente, o mosaico gerado foi armazenado em um SIG (Arc View 3.3) e interpretado a partir da análise visual do produto realçado, utilizando como padrões os elementos tom/cor, textura, forma, tamanho, contexto, geometria e configuração da rede de drenagem. 0 mapeamento dos manguezais foi baseado no reconhecimento de campo realizado em áreas testes na planície costeira do Pará (Mosqueiro, Salinópolis e Bragança) e do Maranhão (Alcântara e São Luís). A identificação desse ecossistema (apenas a floresta de mangue), em função de sua resposta nas imagens Landsat, foi realizada em escala de 1:100.000 e seguiu a classificação proposta pelo ITC para levantamentos geomorfológicos, onde o reconhecimento das formas costeiras está baseado no padrão sedimentar, estratigráfico e mor- 


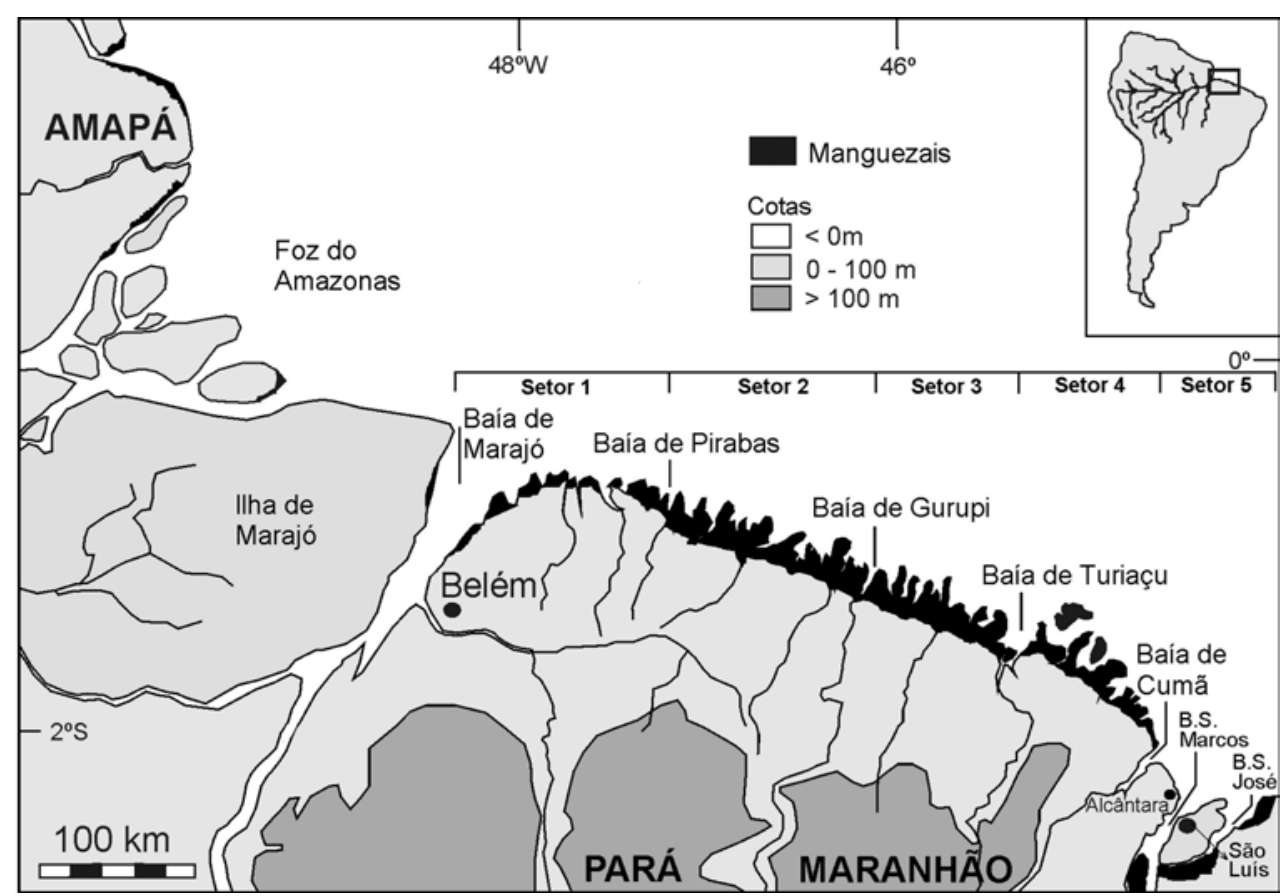

Figura 1 - Mapa de localização da Costa de Manguezais de Macromaré da Amazônia (CMMA)

Tabela 1 - Áreas de manguezal estimadas por continente.

\begin{tabular}{|l|c|c|c|}
\hline \multicolumn{1}{|c|}{ Regiões } & $\begin{array}{c}\text { Área de manguezal }\left(\mathrm{km}^{2}\right) \\
\text { Spalding et al., } 1997\end{array}$ & $\begin{array}{c}\text { Área de manguezal }\left(\mathrm{km}^{2}\right) \\
\text { IUCN, 1983 }\end{array}$ & $\begin{array}{c}\text { Área de manguezal }\left(\mathrm{km}^{2}\right) \\
\text { Fisher \& Spalding, } 1993\end{array}$ \\
\hline Sul e sudeste da Ásia & $75.173(41,5 \%)$ & $51.766(30,7 \%)$ & $76.226(38,3 \%)$ \\
\hline Australásia & $18.789(10,4 \%)$ & $16.980(10 \%)$ & $15.145(7,6 \%)$ \\
\hline Américas & $49.096(27,1 \%)$ & $67.446(40 \%)$ & $51.286(25,8 \%)$ \\
\hline África Oriental & $27.995(15,5 \%)$ & $27.110(16 \%)$ & $49.500(24,9 \%)$ \\
\hline África Ocidental e Oriente Médio & $10.024(5,5 \%)$ & $5.508(3.3 \%)$ & $6.661(3,4 \%)$ \\
\hline Área total & 181.077 & 168.810 & 198.818 \\
\hline
\end{tabular}

Tabela 2 - Áreas de manguezal estimadas por país e o percentual em relação à área total de manguezal no mundo.

\begin{tabular}{|l|c|}
\hline \multicolumn{1}{|c|}{ Países } & $\begin{array}{c}\text { Área de manguezal - } \mathrm{km}^{2} \\
\text { Spalding et al., 1997 }\end{array}$ \\
\hline Indonésia & $42.550(23,5 \%)$ \\
\hline Brazil & $13.400(7,4 \%)$ \\
\hline Austrália & $11.500(6,4 \%)$ \\
\hline Nigéria & $10.515(5,8 \%)$ \\
\hline Cuba & $7.848(4,33 \%)$ \\
\hline Papua Nova Guiné & $5.399(2,94 \%)$ \\
\hline México & $5.315(2,93 \%)$ \\
\hline Total (em relação ao mundo) & $96.527(53,3 \%)$ \\
\hline
\end{tabular}


fológico (Verstappen \& Van Zuidam, 1991). Por fim, as áreas de manguezais foram digitalizadas, suas extensões calculadas e as forçantes que controlam seu desenvolvimento analisadas e discutidas.

\section{RESULTADOS E DISCUSSÃO Compartimentação geomorfológica e mapeamento da CMMA}

A integração com dados de campo das informações obtidas a partir da análise das imagens de sensoriamento remoto (Landsat-7 ETM $^{+}$) permitiu a compartimentação da CMMA em cinco setores geomorfológicos, bem como o mapeamento e quantificação das áreas de manguezais.

0 Setor 1 estende-se da Baía de Marajó até a de Pirabas. Neste, o planalto costeiro alcança a linha de costa, onde se formam falésias sustentadas por sedimentos terciários das formações Barreiras e Pirabas, as quais estão sujeitas à ação de ondas e correntes de marés. A planície costeira é restrita, com menos de $2 \mathrm{~km}$ de largura, e os canais estuarinos estendem-se por $\sim 60 \mathrm{~km}$. Baseado em análise no SIG, a floresta de manguezal ocupa uma área de $842,81 \mathrm{~km}^{2}$ (Fig. 2).

0 Setor 2 está situado entre as baías de Pirabas e Gurupi. Neste, 0 ecossistema de manguezal começa a se desenvolver amplamente a partir da baía de Pirabas e o planalto costeiro recua em direção a sul para constituir falésias inativas. Os manguezais desenvolvidos em direção ao mar atingem atualmente uma largura de $\sim 30 \mathrm{~km}$ e os estuários estendem-se por $\sim 80 \mathrm{~km}$ em direção ao continente. Neste setor os manguezais abrangem uma área de $1.333,97 \mathrm{~km}^{2}$ (Fig. 2).

0 Setor 3 estende-se da Baía de Gurupi até a Baía de Turiaçu. Comparados com os demais, os manguezais nele alcançam sua extensão máxima, com até $\sim 40 \mathrm{~km}$ de largura, formando uma costa muito mais irregular e endentada, provavelmente devido às variações de macromarés. Os manguezais ocupam aqui uma área de $1.846,41 \mathrm{~km}^{2}$ (Fig. 3).

0 Setor 4 está situado entre as baías de Turiaçu e de Cumã. Neste, os manguezais são mais restritos, com largura de $\sim 26 \mathrm{~km}$. A Baía de Cumã marca 0 início de uma pequena interrupção de $\sim 8 \mathrm{~km}$ no cinturão de manguezais. Em direção a leste, a península adjacente de Alcântara é caracterizada por falésias ativas, com aproximadamente $\sim 10 \mathrm{~m}$ de altura, esculpidas na Formação Itapecuru e diretamente limitadas por planícies arenosas de maré, sem significante desenvolvimento de manguezais. Com um todo, neste setor são observados $1.945,01 \mathrm{~km}^{2}$ de manguezal (Fig. 3).
0 Setor 5 estende-se entre a Baía de Cumã e a Ponta de Tubarão (margem leste da Baía de São José). Este setor geomorfológico é representado principalmente pelo ecossistema de pântanos; no entanto os manguezais ocorrem ao longo das margens dos canais estuarinos nas baías de São Marcos e São José (Fig. 4). Os vales estuarinos desenvolvem-se por mais de $100 \mathrm{~km}$ em direção ao continente, onde os manguezais ocupam uma área de $1.622,91 \mathrm{~km}^{2}$. Neste setor, os manguezais voltam a ocorrer somente no sistema estuarino, como na Baía de Marajó. Há, portanto, uma zona de transição da CMMA com a Baía de Marajó, a oeste, e com 0 campo de dunas dos Lençóis Maranhenses, a leste.

\section{Quantificação das áreas de manguezais da CMMA}

A quantificação das áreas de manguezais, utilizando imagens Landsat-7 ETM ${ }^{+}$e um SIG, forneceu valores diferentes daqueles obtidos por outros autores, conforme pode ser observado na Tabela 3. A grande discrepância observada nas quantificações obtidas para o Estado do Pará deve-se ao fato de que, neste trabaIho apenas os manguezais da costa nordeste do Pará que constituem a CMMA foram considerados, enquanto que os resultados de Kjerfve \& Lacerda (1993), oriundos de uma comunicação pessoal de Fearside em 1990, incluem os manguezais pertencentes ao Estado como um todo, em especial os da llha de Marajó.

0 mapeamento dos manguezais a partir da interpretação das suas respostas em imagens Landsat-7 ETM ${ }^{+}$e de um SIG, permitiu a quantificaçãao das áreas de ocorrência na CMMA. 0 valor total de $7.591,09 \mathrm{~km}^{2}$ representa aproximadamente a superfície total de manguezais observados no quinto país com maior extensão desse ecossistema no planeta: Cuba, com $7.848 \mathrm{~km}^{2}$, ou seja, 4,33\% do total no globo. Em relação às Américas, corresponde a 15,5\% dos manguezais existentes. Quanto ao Brasil, com seus $9.200 \mathrm{~km}$ de linha de costa, a CMMA, que ocupa menos de $10 \%$ do litoral do país, abriga $56,6 \%$ do total de manguezais. Por isso, tal setor da costa brasileira é considerado de extrema importância para a conservação da biodiversidade, segundo o Ministério de Meio Ambiente (MMA, 2002).

A fim de explicar as causas da notável ocorrência dos manguezais na CMMA, podemos afirmar que o desenvolvimento deste ecossistema é controlado pela interação continente-oceano, e que sua expansão é determinada pela topografia da superfície préholocênica, bem como pelas condições de energia de ondas e de correntes de marés, como citado por Woodroffe (1992), na costa australiana. Portanto, fatores geomorfológicos, como a grande extensão da plataforma continental adjacente à CMMA, 

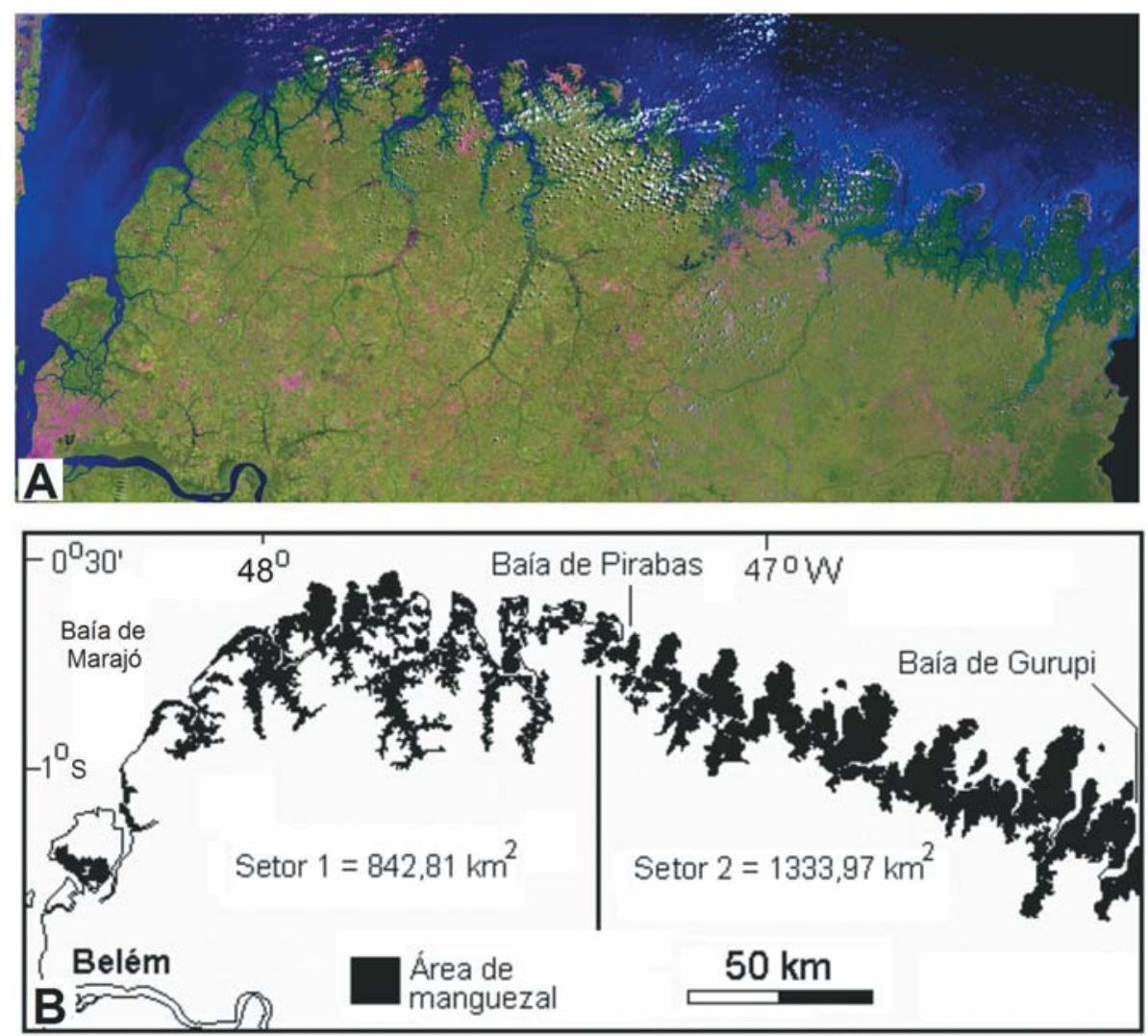

Figura 2 - A) Mosaico de imagens Landsat-7 ETM+ (composição 4R5G3B) mostrando os setores geomorfológicos 1 e 2 da CMMA, ao longo da costa nordeste do Estado do Pará. B) Mapa das áreas de manguezal extraído do mosaico Landsat-7 ETM+ em um SIG.

Tabela 3 - Áreas de manguezal estimadas por estados da costa norte do Brasil e da CMMA.

\begin{tabular}{|c|c|c|c|}
\hline & $\begin{array}{c}\text { Área de manguezal }-\mathrm{km}^{2} \\
\text { Este artigo }\end{array}$ & $\begin{array}{c}\text { Área de manguezal }-\mathrm{km}^{2} \\
\text { Herz, 1991 }\end{array}$ & $\begin{array}{c}\text { Área de manguezal }-\mathrm{km}^{2} \\
\text { Kjerfve and Lacerda, 1993 }\end{array}$ \\
\hline Amapá & - & 1.623 & 1.823 \\
\hline Pará & $2.176,78$ & 1.820 & 3.894 \\
\hline Maranhão & $5.414,31$ & 4.923 & 5.000 \\
\hline CMMA & $7.591,09$ & 6.743 & 8.894 \\
\hline
\end{tabular}

com largura de aproximadamente $200 \mathrm{~km}$, assim como os processos geológicos associados às variações relativas do nível do mar (Cohen et al., 2005), têm permitido, ao longo do Holoceno, 0 desenvolvimento de uma ampla planície costeira, que chega a atingir 40 km de largura. Além disso, podemos citar as condições oceanográficas e meteorológicas favoráveis ao desenvolvimento dos manguezais na área em estudo, como variações de maré entre 4 e $8 \mathrm{~m}$, precipitações superiores a $2.000 \mathrm{~mm}$ anuais e temperaturas médias superiores a $25^{\circ} \mathrm{C}$.

\section{CONCLUSÕES}

A quantificação dos manguezais da CMMA, a partir de dados de imagens Landsat-7 ETM ${ }^{+}$, com pixel reamostrado para $90 \mathrm{~m}$, totalizou uma área de 7.591,09 km². Tal resultado, baseado em uma análise de imagens de resolução espacial moderada realizada no contexto de um SIG, representa um avanço na quantificação das áreas de manguezais do Brasil, particularmente na Amazônia. Essa metodologia pode ser aplicada em outros setores da costa 

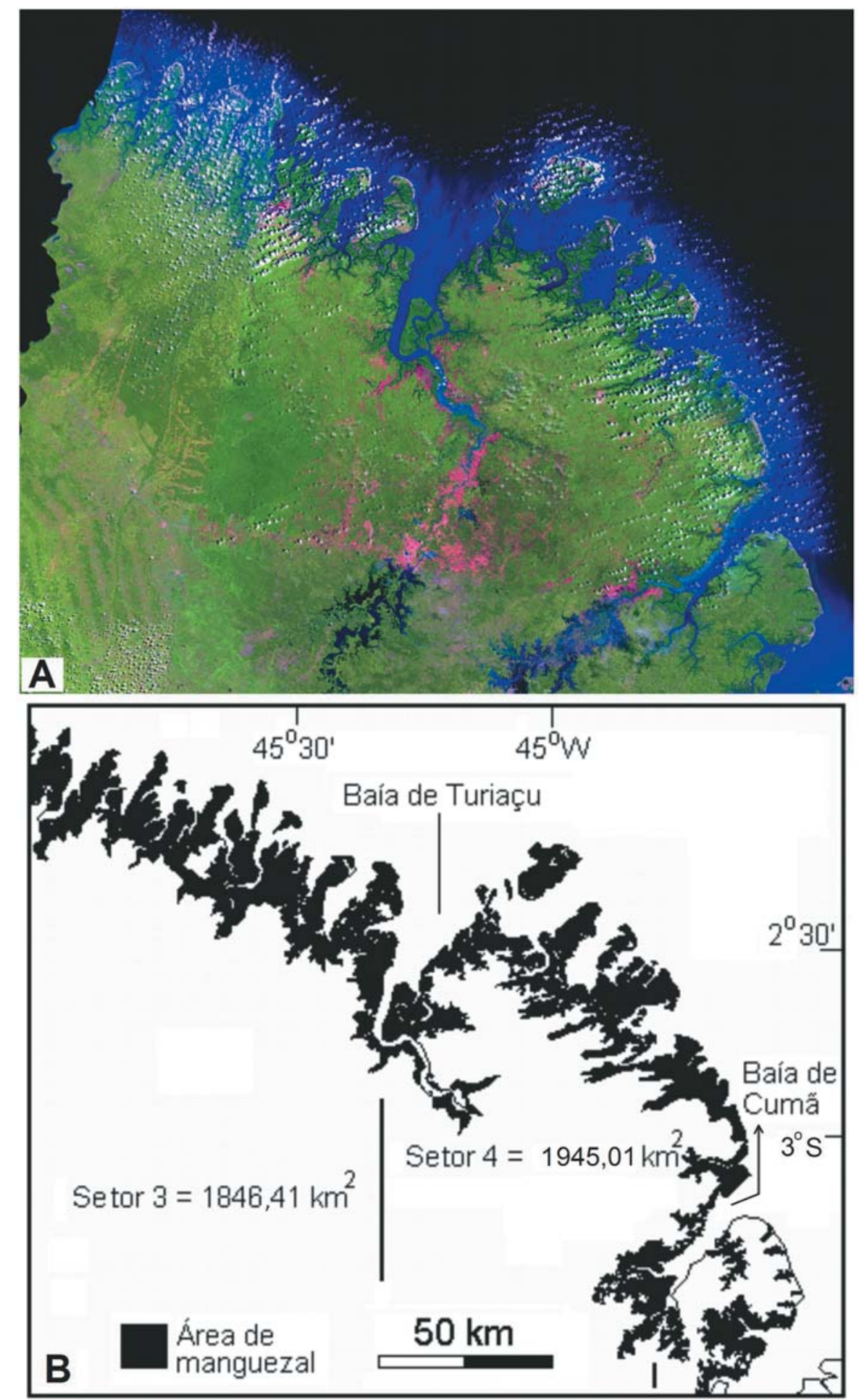

Figura 3 - A) Mosaico de imagens Landsat-7 ETM+ (composição 4R5G3B) mostrando os setores geomorfológicos 3 e 4 da CMMA, ao longo da costa noroeste do Estado do Maranhão. B) Mapa das áreas de manguezal extraído do mosaico Landsat-7 ETM+ em um SIG. 

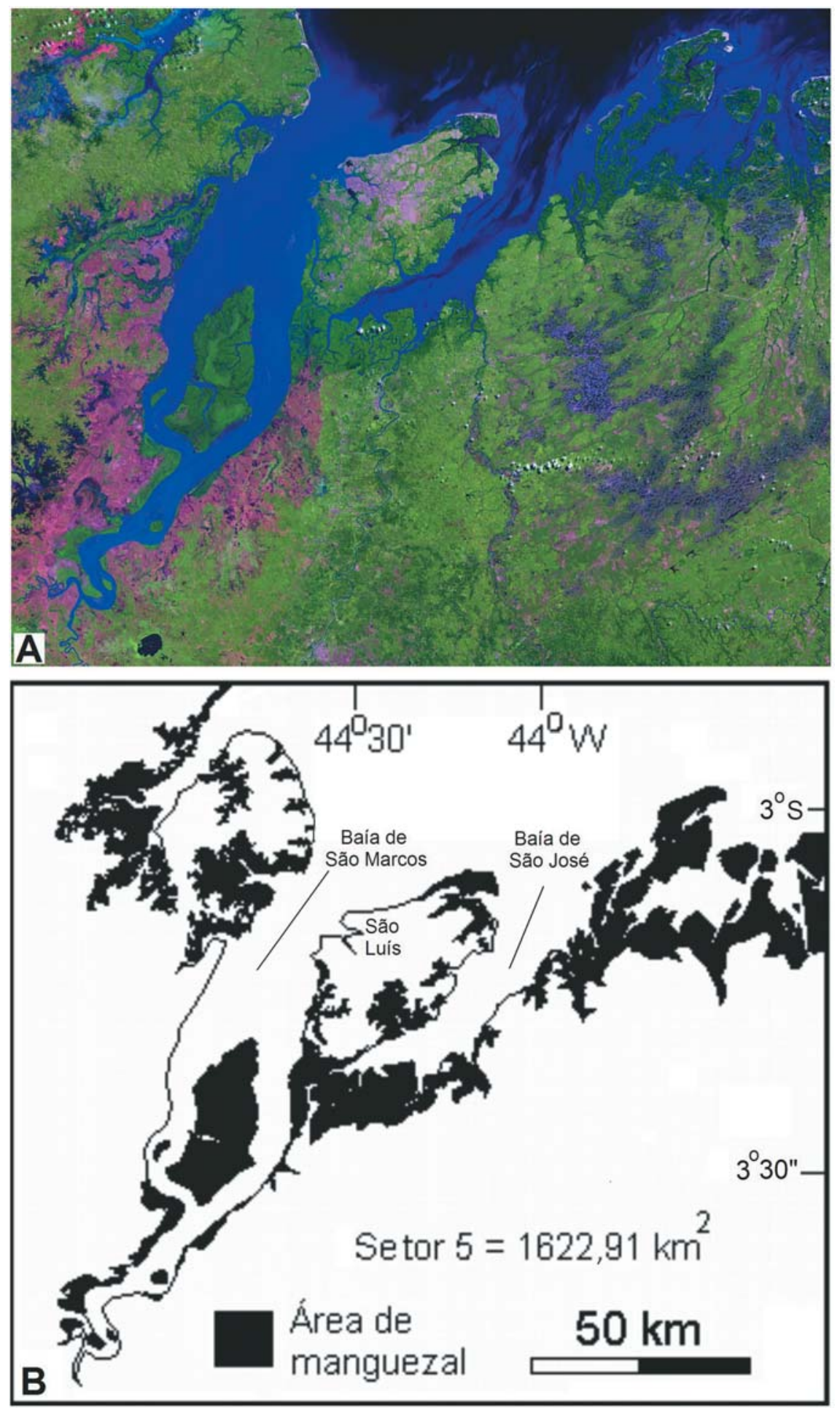

Figura 4 - A) Mosaico de imagens Landsat-7 ETM+ (composição 4R5G3B) mostrando o setor geomorfológico 5 da CMMA, ao longo do Golfão Maranhense (baías de São Marcos e de São José). B) Mapa das áreas de manguezal extraído do mosaico Landsat-7 ETM+ em um SIG. 
brasileira, sempre conjugada à realização de trabalhos de campo para validação dos dados de sensores remotos.

Segundo estimativas disponíveis na literatura, cerca de 50\% das áreas de manguezais de todo o mundo já foram perdidas em função da ocupação desordenada da zona costeira (Kelleher et al., 1995). Assim, o desenvolvimento adequado das pesquisas em zonas costeiras inclui diversos desafios, como o mapeamento em diferentes escalas temporais e espaciais, bem como estabelecimento de programas de monitoramento (Talley et al., 2003).

Medidas prioritárias de conservação da Costa de Manguezais de Macromaré da Amazônia (CMMA) devem ser tomadas e pesquisas que busquem a melhor compreensão deste complexo e importante ecossistema devem ser desenvolvidas. Com efeito, os ecossistemas costeiros amazônicos integram a maior costa de manguezais contínuos do planeta e são considerados de extrema importância biológica de acordo com a "Avaliação e Ações Prioritárias para Conservação da Biodiversidade das Zonas Costeira e Marinha" (MMA, 2002).

\section{AGRADECIMENTOS}

Este trabalho foi financiado pelo projeto PIATAM mar, com apoio da Petróleo Brasileiro S.A. (Petrobras), do Conselho Nacional de Desenvolvimento Científico e Tecnológico (CNPq Proc. \#502450/03-7) e do Projeto Rede 05 / Petromar /Petrorisco (CtPetro/FINEP/Petrobras/CNPq). 0 autor agradece ao CNPq pelo auxílio à pesquisa durante esta investigação (Proc. \#303238/2002-0). Esta é a contribuição de ño 4 do Projeto PIATAM mar.

\section{REFERÊNCIAS}

CLOUGH BF. 1993. The economic and environmental values of mangrove forest and their present state of conservation in the south-east Asia/Pacific region. Mangrove Ecosystem Technical Report No. 1. ITTO/ISME, Okinawa, 202 pp.

COHEN MCL, SOUZA FILHO PWM, LARA RJ, BEHLING H \& ANGULO RJ. 2005. A model of Holocene mangrove development and relative sealevel changes on the Bragança Peninsula (Northern Brazil). Wetlands Ecology And Management, 13(4): 433-443.

DHN - DEPARTAMENTO DE HIDROGRAFIA E NAVEGAÇÃO. 2004. Tábuas de marés para 1994. Costa do Brasil e alguns portos estrangeiros. DHN, Rio de Janeiro, 1-6.

DIOP ES. 1993. Conservation and sustainable utilization of mangrove forest in Latin America and Africa regions. Part II - Africa. Mangrove Ecosystem Technical Report No. 3. ITTO/ISME, Okinawa, 262 pp.
FISHER P \& SPALDING MD. 1993. Protected Areas with Mangrove Habitat. Draft Report. World Conservation Monitoring Centre, Cambridge, UK. 60 pp.

FRANZINELLIE. 1992. Evolution of the geomorphology of the coast of the State of Pará, Brazil. In: Évolution des littoraux de Guyane et de la Zone Caraïbe Méridionale pendant le Quaternaire. PROST MT (Ed.). ORSTOM, Paris, 203-230.

HERZ R. 1991. Manguezais do Brasil. São Paulo. Instituto Oceanográfico, Universidade de São Paulo. 227 pp.

IUCN. 1983. Global Status of Mangrove Ecosystems. Commission on Ecology Papers No. 3. International Union for Conservation of Nature and Natural Resources, Gland, Switzerland. $88 \mathrm{pp}$.

KELLEHER G, BLEAKLEY C \& WELLS S. 1995. A global representative system of marine protected area. Volume 1, World Bank, Washington D.C. $230 \mathrm{pp}$.

KJERFVE B \& LACERDA LD. 1993. Mangroves of Brazil. In: Conservation and sustainable utilization of mangrove forest in Latin America and Africa regions. Part I - Latin America, LACERDA LD (Ed.). Mangrove Ecosystem Technical Report No. 2. ITTO/ISME, Okinawa, 272 pp.

KJERFVE B, PERILLO GME, GARDNER LR, RINE JM, DIAS GTM \& MOCHEL FR. 2002. Morphodynamics of muddy environments along the Atlantic coasts of North and South America. In: Muddy Coasts of the World: Processes, Deposits and Functions. Amsterdam, HEALY TR, WANG Y \& HEALY J-A (Ed.). Elsevier, N.Y., 479-532.

LACERDA LD. 1993. Conservation and sustainable utilization of mangrove forest in Latin America and Africa regions. Part I - Latin America. Mangrove Ecosystem Technical Report No. 2. ITTO/ISME, Okinawa, $272 \mathrm{pp}$.

MMA - Ministério do Meio Ambiente. 2002. Avaliação e Ações Prioritárias para Conservação da Biodiversidade das Zonas Costeira e Marinha. MMA/SBF, Brasília, 72 pp.

MORAES BC, COSTA JMN \& COSTA ACL. 2005. Variação espacial e temporal da precipitação no estado do Pará. Acta Amazônica, 35: 207214.

PROST MT. 1997. La mangrove de front de mer en Guyane: ses transformations sous l'influence du système de dispersion Amazonien et son suivi par télédétection. In: Mangrove ecosystem studies in Latin America and Africa, KJERFVE B, LACERDA LD \& DIOP EHS (Ed.). UNESCO, Paris, 111-126.

RABUS B, EINEDER M, ROTH A \& BAMLER R. 2003. The shuttle radar topography mission - a new class of digital elevation models acquired by spaceborne radar. ISPR Journal of Photogrammetry \& Remote Sensing, 57: 241-262.

REBELO-MOCHEL F. 1997. Mangroves on São Luís Island, Maranhão Brazil. In: Mangrove ecosystem studies in Latin America and Africa, KJERFVE B, LACERDA LD \& DIOP EHS (Ed.). UNESCO, Paris, 145-154. 
SCHAEFFER-NOVELLI Y. 1989. Perfil dos ecossistemas litorâneos brasileiros, com especial ênfase sobre o ecossistema manguezal. Pub. Esp. do Instituto Oceanográfico, 7: 1-16.

SCHAEFFER-NOVELLI Y, CINTRÓN-MOLERO G, ADAIMA RR. 1990. Variability of mangrove ecosystems along the Brazilian coast. Estuaries, 13: 204-218.

SCHAEFFER-NOVELLI Y \& CINTRÓN-MOLERO G. 1999. Brazilian mangroves: a historical ecology. Jornal Ciência e Cultura, 51: 274-286.

SPALDING M, BLASCO F \& FIELD C. 1997. World mangrove atlas. ISME, Okinawa, 178 pp.
TALLEY DM, NORTH EW, JUHL AR, TIMOTHY DA, BROWER JFC, BROWN CA, CHASAR LC, HALL CJJ, MEYSMAN F, NEMERSON DM, SOUZA FILHO PWM \& WOOD RJ. 2003. Research Challenges at the land-sea interface. Estuarine Coastal and Shelf Science, 58: 699-702.

VERSTAPPEN HT \& VAN ZUIDAM RA. 1991. The ITC system of geomorphologic survey. ITC Publication n. 10, Netherlands, 89 pp.

WOODROFFE C. 1992. Mangrove sediments and morphology. In: Tropical mangrove ecosystems, ROBERTSON AI \& ALONGI DM (Ed.). AGU, Washington, 7-41.

\section{NOTA SOBRE O AUTOR}

Pedro Walfir Martins Souza Filho. Professor Doutor do Departamento de Geologia do Centro de Geociências da UFPA desde 2002. Desenvolveu mestrado na área de geologia costeira e doutorado na área de sensoriamento remoto aplicado à geologia costeira no Programa de Pós-Graduação em Geologia e Geoquímica da UFPA. É bolsista de produtividade em pesquisa nível II do CNPq desde 2003 e atualmente coordena o Laboratório de Análise de Imagens do Trópico Úmido do Centro de Geociências da UFPA. 